\title{
Modern trends in GI-oncology imaging
}

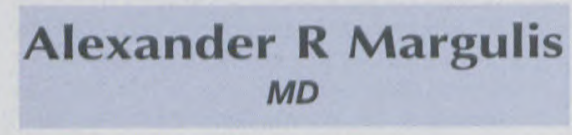

\section{Historical highlights}

Gastrointestinal Imaging has undergone dramatic changes since its inception. Barium double contrast upper and lower alimentary tube examinations have achieved a high degree of technical excellence in the seventies. Enteroclysis emerged in the eighties as a sophisticated approach for examining the small bowel. CT and MRI came on the scene in the late eighties and continued in the nineties as fruitful approaches for studying acute conditions affecting the GI tube. It was only in the late nineties with the advent of the spiral (helical) and then multi row detector CT that cross-sectional imaging became a serious competitor to barium studies. Virtual endoscopy is about to become clinical and optical approaches will be upon us before we know it.

Barium imaging has undergone great changes during the last 20 years. Its use after a glorious era of superb double contrast artistry has gradually decreased. Endoscopy has advanced, become more patient friendly and as it has the advantages of biopsy and self-referral it has practically eliminated the barium studies of the upper and lower alimentary tube in the industrialized world. Lately cross-sec- tional imaging, particularly multi row detector CT has taken away many of its applications. As barium examinations are used less frequently in most radiology training programs, the number of examinations is insufficient for adequate training. The quality of barium examinations and their accuracy is therefore generally decreasing for most old favorites, such as the double contrast barium enema and the upper gastrointestinal examination.

\section{Present uses of barium sulfate}

Is barium dead? It is not. It has just lost its preeminence and has metamorphosed. Some applications have survived. Barium is still supreme for some examinations where fluoroscopy and an intraluminal contrast medium give immediate and accurate answers in many clinical situations. To name the most important:

1. To study motility.

2. Pre surgery for reflux remedy.

3. To evaluate the size of hiatus hernia.

4. To find the site of suspected perforation. (After iodine contrast medium fails to show site, barium is used as it has better signal to noise.)

5. Same applies for localizing gastrostomy or jejunostomy tubes, (if the iodine study fails to give acceptable answers).

6. Same applies to find or exclude the presence of anastomotic leaks.

7. For small bowel studies, including double contrast enteroclysis.

8. As an additive to avoid using the rigorous cleansing maneuvers inherited from barium enema and conventional colonoscopy, before performing $\mathrm{CT}$ or $\mathrm{MR}$ virtual colonoscopy.

9. When conventional endoscopy cannot be performed or has failed for whatever reason.

Some contrast media that are inexpensive, safe and have been around for many years just refuse to die.

\section{Present role of cross-sectional imaging}

The increasing computer power in cross-sectional imaging and particularly the advent of multi row detector CT has for the first time facilitated the acquisition of 3 dimensional data, permitting high resolution volumetric acquisition of images, thus facilitating diagnosis. Multi row detector CT making 3D acquisition possible, and 3D MR have made virtual endoscopy an increasingly accepted clinical imaging technique. This technique is presently being routinely used in the colon and is increasingly replacing barium enemas as well as diagnostic conventional colonoscopy.

Virtual CT endoscopy is starting to be applied to practically every part of the GI tube: esophagus, stomach, small bowel, mesenteric blood vessels, although these procedures are still in the research stage.

MR Imaging of the alimentary tube is very promising. It has with fast sequences been able to suppress artifacts produced by peristalsis and with 
its wide range of soft tissue contrast resolution has been very informative particularly in the evaluation of Crohn's disease, pelvic lipomatosis and mesenteritis. It also shows promise in the assessment of cancer of the entire alimentary tube.

MR virtual colonoscopy has not as yet achieved the general acceptance of its CT equivalent, but it is gaining acceptance in several European centers. It is even being used as part of MR total body screening programs.

Different approaches are also being tested to eliminate the need for bowel cleansing before the performance of MR colonoscopy, one using gadolinium enema the other adding barium to a low residue diet for several days before the examination.

\section{PET- CT}

Fusion of images generated from different imaging modalities, such as $\mathrm{MR}, \mathrm{CT}$ and PET is showing that advantages of two techniques can be maximized. This approach has great potential in oncologic imaging of the alimentary tube. The advantages of PET's ability to detect malignant nodes are combined with the superior spatial resolution of CT. This is of particular value in the staging of carcino$\mathrm{ma}$ of the esophagus where the evaluation of mediastinal nodes is essential.

\section{Computer-aided detection (CAD)}

The reduction and eventually the elimination of perception errors in cross-sectional studies of the alimentary tube will be of particular importance in screening with CT virtual colonoscopy. The present approaches use neural-network computers that are taught what is normal with all its variations. The computer is the able to distinguish polyps and cancer from folds, although different techniques will be necessary for the distinction from fecal residue. $\mathrm{CAD}$ should also help in providing screening at an affordable price. Computer aided diagnosis will be particularly important in the studies of populations at risk as identified in the future with genetic screening. Virtual colonoscopy screening of such populations will improve the odds of survival.

\section{Optical coher- ence tomography}

The development of new imaging modalities like optical coherence tomography, adds new dimensions to medical imaging of the alimentary tube. It is expected that it will be possible in the future to identify early precancerous, dysplastic, changes in many parts of the GI tube. Examples are changes in Barrett's esophagus as well as in the colon mucosa in chronic ulcerative colitis.

\section{Molecular imaging of the alimentary tube}

At present this approach is still only experimental. At this time the most successful approach consists of introducing exogenous reporter genes into cancer cells. These are then implanted into the peritoneal cavity of nude mice with either fluorescent, (optical) or radioactive probes. It is probable that such approaches will be soon introduced into clinical practice.

\section{Suggested reading}

1. Horton KM, Fishman EK. Volume-rendered 3D CT of the Mesenteric Vasculature: Normal Anatomy, Anatomic Variants, and Pathologic Conditions. Radiographics 2002; 22: 161-172.

2. Johnson CD, Dachman AH. CT Colonography: The Next Colon Screening Examination? Radiology 2000; 216: 331-341.

3. Ferrucci, JT. Colon Cancer Screening with Virtual Colonoscopy. AJR 2001; 177: 975-988.

4. Luboldt W, Bauerfeind P, Wildermuth S, Marincek B, Fried M, Debatin JF. Colonic Masses: Detection with MR Colonography. Radiology 2000; 216.

5. Luboldt W, Morrin MM. MR colonography: status and perspective. Abdoml Imaging 2002; 27: 400-409.

6. Tearney GI, Brezinski ME, Southern JF, Bouma BE, Boppart SA, Fujimoto JG. Optimal Biopsy in Human Gastrointestinal Tissue Using Optical Coherence Tomography. The American Journal of Gastroenterology 1997; 92: 1800-1804.

7. Lander ES, et al. Initial sequencing and analysis of the human genome. Nature 2001; 409: 814823.

8. Venter $\mathrm{JC}$, et al. The sequence of the human genome. Science 2001; 291: 1304-1351.

9. Hillman BJ, Neiman HL. Translating Molecular Imaging Research into Practice; summary of the Proceedings of the American college of Radiology Colloquium, April 22-24, 2001 Radiology 2002; 222: 19-24. 